\title{
The TRENDS High-contrast Imaging Survey. VII. Discovery of a Nearby Sirius-like White Dwarf System (HD 169889)
}

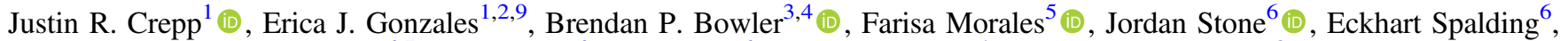 \\ Amali Vaz ${ }^{6,7}$, Philip Hinz ${ }^{6}$, Steve Ertel $^{6}$, Andrew Howard ${ }^{4}$, and Howard Isaacson ${ }^{8}$ (i) \\ ${ }^{1}$ Department of Physics, University of Notre Dame, 225 Nieuwland Science Hall, Notre Dame, IN 46556, USA; jcrepp@nd.edu \\ ${ }^{2}$ Department of Astronomy, University of California, Santa Cruz, 1156 High Street, Santa Cruz, CA 95064, USA \\ ${ }^{3}$ Department of Astronomy, The University of Texas at Austin, TX 78712, USA \\ ${ }^{4}$ Department of Astronomy, California Institute of Technology, 1200 E. California Boulevard, Pasadena, CA 91125, USA \\ 5 Jet Propulsion Laboratory, 4800 Oak Grove Drive, Pasadena, CA 91109, USA \\ ${ }^{6}$ Steward Observatory, Department of Astronomy, University of Arizona, 933 N. Cherry Avenue, Tucson, AZ 85721, USA \\ ${ }_{7}^{7}$ Large Binocular Telescope, Mount Graham International Observatory, Safford, AZ 85546, USA \\ ${ }^{8}$ Department of Astronomy, University of California, Berkeley, CA 94720, USA \\ Received 2018 May 7; revised 2018 July 11; accepted 2018 July 11; published 2018 August 29
}

\begin{abstract}
Monitoring the long-term radial velocity (RV) and acceleration of nearby stars has been proven as an effective method for directly detecting binary and substellar companions. Some fraction of nearby RV trend systems are expected to be comprised of compact objects that likewise induce a systemic Doppler signal. In this paper, we report the discovery of a white dwarf (WD) companion found to orbit the nearby ( $\pi=28.297 \pm 0.066$ mas) G9 V star HD 169889. High-contrast imaging observations using NIRC2 at the W. M. Keck Observatory and LMIRCam at the Large Binocular Telescope (LBT) Observatory uncover the $\left(\Delta H=9.76 \pm 0.16, \Delta L^{\prime}=9.60 \pm 0.03\right)$ companion at an angular separation of 0 ." 8 (28 au). Thirteen years of precise Doppler observations reveal a steep linear acceleration in the RV time series and place a dynamical constraint on the companion mass of $M \geqslant 0.369 \pm 0.010 M_{\odot}$. This "Sirius-like" system adds to the census of WD companions suspected to be missing from surveys of in the solar neighborhood.
\end{abstract}

Key words: astrometry - stars: individual (HD 169889, HIP 90365, SAO 123479) - techniques: high angular resolution - techniques: radial velocities - white dwarfs

\section{Introduction}

Many undiscovered white dwarf (WD) companions remain hidden in the solar neighborhood, their faint signals overwhelmed by the glare of light emitted from a nowbrighter unevolved companion star at optical and infrared wavelengths. The occurrence of these "Sirius-like" systemsbinaries with a WD orbiting a solar-type or earlier-type starrepresents only $\approx 8 \%$ of known WDs in close proximity to the Sun; this rate falls precipitously at distances beyond $\approx 25 \mathrm{pc}$ due to observational bias (Holberg et al. 2013). WD companions are routinely missed from seeing-limited surveys that are relegated to studying large projected separations (Parsons et al. 2016).

Directly imaged WDs in Sirius-like systems are valuable benchmark objects that confer information regarding empirical mass-radius relations, the luminosity function of WDs from precise parallax measurements of the host star, calibration of progenitor mass relations, atmospheric characterization and chemical constituents, and cooling timescales based on age estimates of the host star (Harris et al. 2006; Kilic et al. 2006; Matthews et al. 2014; Holberg et al. 2016; Bacchus et al. 2017; Joyce et al. 2017; Parsons et al. 2017).

Hubble Space Telescope imaging follow-up measurements based on the presence of excess UV flux has been shown as a reliable method to identify Sirius-like WD companions (Parsons et al. 2016). At near-infrared wavelengths, groundbased adaptive optics (AO) observations of Sun-like stars can

\footnotetext{
${ }^{9}$ NSF Graduate Research Fellow.
}

also be employed. Given that a blind survey (that is uninformed by dynamical signposts or other means) to detect WD companions would be inefficient, long-term radial velocity (RV) monitoring of Sun-like stars can be used instead to reveal the presence of compact objects as their orbits evolve over timescales of years to decades (Crepp et al. 2014; Rodigas et al. 2016). Orbital monitoring through imaging and precise RV measurements further permits studies of the period and eccentricity distribution of WD companions as currently only a handful have complete orbits (Bond et al. 2017). The orbital architecture of WD binaries informs studies that relate orbital evolution to the loss of companions (the rate of which is predicted to be significant at short orbital periods), circumstellar disks, mass transfer, and type Ia supernovae explosions (Parsons et al. 2016).

In this paper, we report the discovery of a Sirius-like WD companion orbiting the nearby $(d=35.34 \pm 0.08 \mathrm{pc})$ Sun-like star HD 169889 (Table 1). The companion was originally mistaken as a brown dwarf candidate based on its brightness and neutral infrared color. However, RV measurements over a time baseline of 13 years combined with AO imaging places a lower limit on the companion mass of $M \geqslant 0.369 \pm 0.010 M_{\odot}$. We describe the observations acquired from Keck and the Large Binocular Telescope (LBT) that detect (Section 2), confirm (Section 3), and provide a provisional characterization of the physical properties of the WD (Sections 4 and 5). HD $169889 \mathrm{~B}$ adds to a small but growing list of compact objects in the local sample that can be studied in detail by inferring properties from its optical host star and understanding their interaction. 


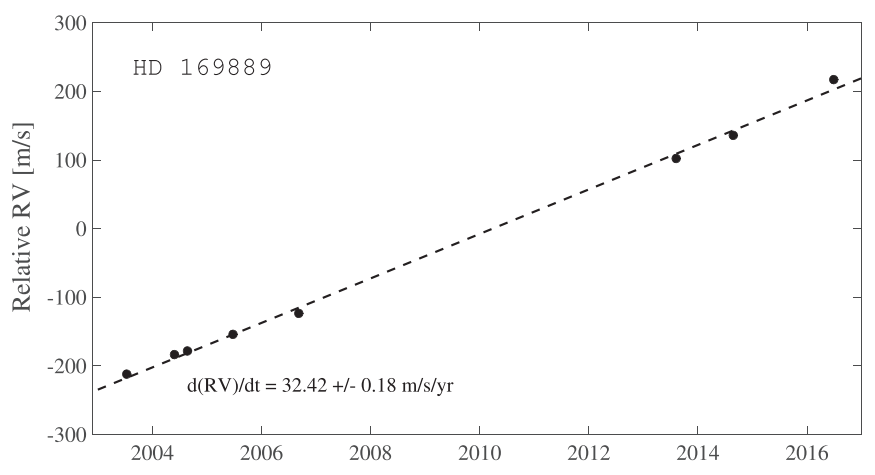

Figure 1. Stellar RV measurements for HD 169889 using HIRES at Keck. A long-term Doppler acceleration indicates the presence of a distant companion. Uncertainties are shown but are too small to be seen at this scale.

\section{Observations}

\subsection{High-resolution Spectroscopy}

Precise stellar RV measurements were obtained as part of the California Planet Search program (Howard et al. 2010a). HD 169889 was observed using the High Resolution Echelle Spectrometer (HIRES) with the Keck I telescope (Vogt et al. 1994). Standard methods for precise Doppler observations were used for spectral calibration, extraction, and RV analysis (Howard et al. 2010b). A total of eight Doppler measurements (relative to an arbitrary zero-point) were recorded from 2003 through 2016 (Table 2); a significant acceleration was noticed within the first several years of RV follow-up. HD 169889 was subsequently observed with a relatively low cadence compared to Doppler searches meant specifically for planets. As with other TRENDS discoveries, visual inspection of RV time series measurements along with numerical investigation of Doppler accelerations led to the identification of HD 169889 as a highpriority target for follow-up high-contrast imaging (Crepp et al. 2012a, 2014).

Fitting the RV slope as a straight line, we find an average acceleration $^{10}$ of $\dot{v}=32.42 \pm 0.18 \mathrm{~m} \mathrm{~s}^{-1} \mathrm{yr}^{-1}$. As can be seen in Figure 1, when comparing a systemic trend to the HIRES measurements a low-level change in the acceleration over time can be seen. While the instantaneous acceleration places a lower limit on the directly imaged companion mass (see Section 4), curvature information (the "jerk", $\ddot{v}$ ) can ultimately be used to constrain eccentricity and other orbital parameters given sufficient imaging and RV data (Crepp et al. 2012b).

\subsection{High-contrast Imaging}

\subsubsection{Keck/NIRC2}

HD 169889 was observed using NIRC2 with the Keck II AO system in natural guide star mode on 2015 May 29 UT, 2015 August 28 UT, and 2016 April 21 UT (Wizinowich et al. 2000). Measurements were obtained at near-infrared wavelengths using the narrow field camera mode $(1024 \times 1024$ pixels with 10 mas plate scale). The field derotator was turned off (vertical angle mode) to enable speckle suppression through angular differential imaging (Marois et al. 2006). Table 3 lists a summary of the observations.

\footnotetext{
${ }^{10}$ Doppler analysis takes into account the velocity offset in 2004 resulting from the HIRES detector upgrade, which is treated as a nuisance parameter through the statistical (Markov Chain Monte Carlo) marginalization process.
}

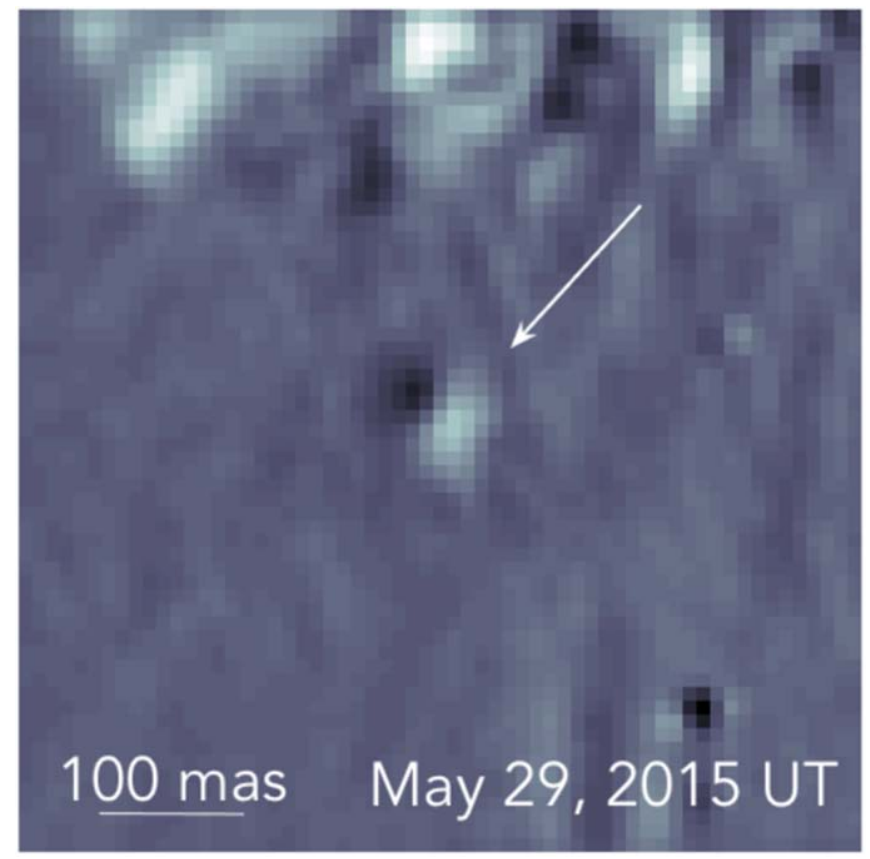

Figure 2. Discovery image of HD 169889 B. Recorded in the $K_{s}$ filter with field minimal rotation, this image provided sufficient motivation to re-observe the star and ultimately confirm the WD companion.

Initially meant to vet the target for binarity with a snapshot imaging sequence, the first observations of HD 169889 acquired in 2015 May resulted in a marginal detection of what would later be confirmed as the optical companion that we refer to as HD 169889 B. Figure 2 shows the dipole pattern which results from a partially subtracted companion point-spread function (PSF) close to the optical host $\operatorname{star}(\theta \approx 832$ mas). Given that the first epoch yielded only coarse astrometry and photometry information, subsequent epochs with larger angular rotation were obtained to avoid self-subtraction and distinguish the object from speckle noise. Follow-up observations were obtained in $H$ and $K_{s}$ with NIRC2 in subsequent observing seasons to assess companion color and relative proper motion. Due to constraints from weather, parallel observing programs, and other logistics, unsaturated images of the optical host star were only obtained in the $H$-band (2016 April) to allow for relative photometry measurements, making the $K_{s}$ data (2015 August, 2016 August) only helpful for astrometry (Section 3). Standard data analysis methods were used to fully process the differential imaging sequence as employed in other TRENDS detections (Crepp et al. 2014, 2016).

\subsection{2. $L B T / L M I R C a m$}

A separate sequence of mid-infrared observations were recorded by LMIRCam on the LBT on 2016 June 20 UT (Skrutskie et al. 2010). With fewer optical reflections than traditional AO systems, LBT offers lower thermal background levels in the mid-infrared. Imaging measurements were obtained using the LBT Interferometer (LBTI) but using only the left side $8.4 \mathrm{~m}$ aperture (Hinz et al. 2016). Since the LBTI instrument has no derotator, all fields are observed to rotate with the parallactic angle on the detector. To track timevariable sky background and detector drifts we nodded the star up and down with a throw of 4 ." 5 every 50 frames. 
Data were reduced using the LEECH-survey pipeline (Skemer et al. 2014). In short, the pipeline implements the following steps. Bad pixels are fixed by replacing their values with the median of the nearest eight functioning pixels. The median of each detector channel is subtracted from the corresponding pixel columns to correct for bias drifts on timescales shorter than nods. Background emission is removed from each image by subtracting the median of 50 images recorded in the opposite nod position taken closest in time. Each image is dewarped using the coefficients reported by Maire et al. (2015). Since LBTI/LMIRCam pixels oversample the single-aperture PSF, we bin each image into $2 \times 2$ pixels, which has the effect of removing any residual bad pixels or cosmic-ray hits. Images are registered using a cross-correlation method and then median-combined into sets of 20 or sets with less than two degrees of rotation (Figure 4). This rotation limit is chosen so that a companion at the edge of the reduced $3^{\prime \prime} \times 3^{\prime \prime}$ field of view will move by less than $\approx 1$ PSF width.

The LEECH pipeline implements a principal component analysis (PCA) to fit and remove the influence of the central star before de-rotating and stacking images (Amara \& Quanz 2012; Soummer et al. 2012). PCA proceeds annulus-byannulus using a width of 9 pixels $(\approx 2 \lambda / D)$ to fit for the starlight and then subtracting the best fit from an annulus only 1-pixel wide. We fit for the optimal number of principal components at each radius by injecting fake planets and iterating until we reach the best contrast. The position and flux of HD 169889 B were fit simultaneously by subtracting a shifted and scaled image of the unsaturated primary star, which we used as a model PSF. This was done for the mediancombined images before the high-contrast data processing steps to properly account for algorithm effective throughput.

We use the spectral-type color relations from Bessell \& Brett (1988) to estimate the $L^{\prime}$ magnitude of HD 169889 A, finding $L^{\prime}=6.44$. This result is consistent with WISE photometry, $W_{1}=6.45 \pm 0.07$, which is comparable to $L^{\prime}$ at this level of precision. The resulting apparent magnitude and absolute magnitude of HD $169889 \mathrm{~B}$ are listed in Table 4.

\section{Astrometry}

HD 169889 has a large proper motion across the sky (Table 1). An astrometric time baseline of 1.2 years (2015 May to 2016 August), which includes five imaging detections, provided unambiguous confirmation that the companion is comoving. We plot the measured position of HD $169889 \mathrm{~B}$ relative to HD $169889 \mathrm{~A}$ and compare to the null-hypothesis that describes the path of a distant background source (Figure 3). ${ }^{11}$

As mentioned previously, the discovery image of HD 169889 B from 2015 May achieved only marginal parallactic angle rotation (Table 3). Nevertheless, we still use astrometry from this epoch. Though the dipole intensity pattern of the companion could not be removed, it can still be modeled by injecting fake companions. Following a procedure similar to that which allows for photometric calibration of self-subtraction, we estimate the position angle (PA) of the companion from this epoch and adopt conservative uncertainties to accommodate systematics from rotational shear (Table 4). All

\footnotetext{
${ }^{11}$ NIRC2 was opened for installation of new filters just prior to the first observation of HD 169889 B. The plate scale, image distortion solutions, and position angle offset from Service et al. (2016) are applicable to all measurements.
}

Table 1

Upper Panel: Observational Parameters of HD 169889 A

\begin{tabular}{|c|c|}
\hline \multicolumn{2}{|c|}{ HD 169889 Properties } \\
\hline R.A. (J2000) & 182621.94 \\
\hline Decl. (J2000) & +083656.74 \\
\hline$B$ (mag) & 9.02 \\
\hline$V(\operatorname{mag})$ & 8.27 \\
\hline$R$ (mag) & 7.8 \\
\hline$I$ (mag) & 7.4 \\
\hline$J$ (mag) & $6.906^{\mathrm{a}}$ \\
\hline$H$ (mag) & $6.560^{\mathrm{a}}$ \\
\hline$K_{s}(\mathrm{mag})$ & $6.486^{\mathrm{a}}$ \\
\hline$\pi$ (mas) & $28.297 \pm 0.066^{\mathrm{b}}$ \\
\hline $\mathrm{d}(\mathrm{pc})$ & $35.34 \pm 0.08^{\mathrm{b}}$ \\
\hline$\mu_{\alpha}\left(\operatorname{mas} \mathrm{yr}^{-1}\right)$ & $-195.671 \pm 0.073^{\mathrm{b}}$ \\
\hline$\mu_{\delta}\left(\operatorname{mas} \mathrm{yr}^{-1}\right)$ & $-469.247 \pm 0.066^{\mathrm{b}}$ \\
\hline Mass $\left(M_{\odot}\right)$ & $0.89 \pm 0.13$ \\
\hline Radius $\left(R_{\odot}\right)$ & $0.88 \pm 0.04$ \\
\hline $\log L\left(L_{\odot}\right)$ & $-0.24 \pm 0.04$ \\
\hline $\log R_{\mathrm{HK}}^{\prime}$ & -4.9 \\
\hline$S_{\text {ave }}$ & 0.19 \\
\hline Gyro Age (Gyr) & $5.2_{-1.5}^{+1.3 \mathrm{c}}$ \\
\hline SME Age (Gyr) & $5.5-12.9$ \\
\hline$[\mathrm{Fe} / \mathrm{H}](\mathrm{dex})$ & $-0.14 \pm 0.01^{\mathrm{d}}$ \\
\hline $\log g\left(\mathrm{~cm} \mathrm{~s}^{-2}\right)$ & $4.49 \pm 0.03^{\mathrm{d}}$ \\
\hline$T_{\text {eff }}(K)$ & $5360 \pm 25^{\mathrm{d}}$ \\
\hline Spectral Type & G9 V \\
\hline$v \sin (i)\left(\mathrm{km} \mathrm{s}^{-1}\right)$ & 0.50 \\
\hline
\end{tabular}

Notes. Lower panel: physical properties are derived from HIRES template spectra and theoretical isochrones (Valenti \& Fischer 2005).

a NIR magnitudes from 2-Micron All Sky Survey (2MASS) catalog of point sources (Cutri et al. 2003; Skrutskie et al. 2006).

b Parallax from Gaia mission DR2 (Gaia Collaboration et al. 2016, 2018).

${ }^{c}$ Gyrochronological age based upon empirical relations (Mamajek \& Hillenbrand 2008).

${ }^{\mathrm{d}}$ Spectral fitting results from Brewer et al. (2016).

other epochs were analyzed self-consistently using the methods described in other TRENDS discoveries (Crepp et al. 2013, 2014).

\section{Companion Mass Limit from Dynamics}

We use the precise stellar RV measurements obtained from HIRES along with imaging follow-up measurements to place a lower limit on the mass of HD 169889 B from dynamics. The eight Doppler measurements are fit with a straight line to estimate the acceleration of the star induced by the companion. We augment the internal RV uncertainties listed in Table 2 with a "jitter" term that is added in quadrature to accommodate the expected level of noise introduced from stellar variability. HD 169889 A has a visual color of $B-V=0.75$ and median activity index of $S_{\mathrm{HK}}=0.18$. We assume a $B-V$ uncertainty of 0.01 mags. Using empirical relations from Isaacson \& Fischer (2010), we estimate a jitter value of $\sigma=2.2 \pm 0.1 \mathrm{~m} \mathrm{~s}^{-1}$.

A straight line is fit using Markov Chain Monte Carlo (MCMC) methods to find a best-fitting RV slope of $\dot{v}=32.42 \pm 0.18 \mathrm{~m} \mathrm{~s}^{-1} \mathrm{yr}^{-1}$. Combined with the direct imaging projected separation, we use the relation from Liu et al. (2002) to derive a lower limit to the companion mass of $m_{\min }=0.369 \pm 0.010 M_{\odot}$ (Table 5). Clearly, HD $169889 \mathrm{~B}$ cannot be a brown dwarf but is instead a compact object provided the acceleration is attributable to this object. 

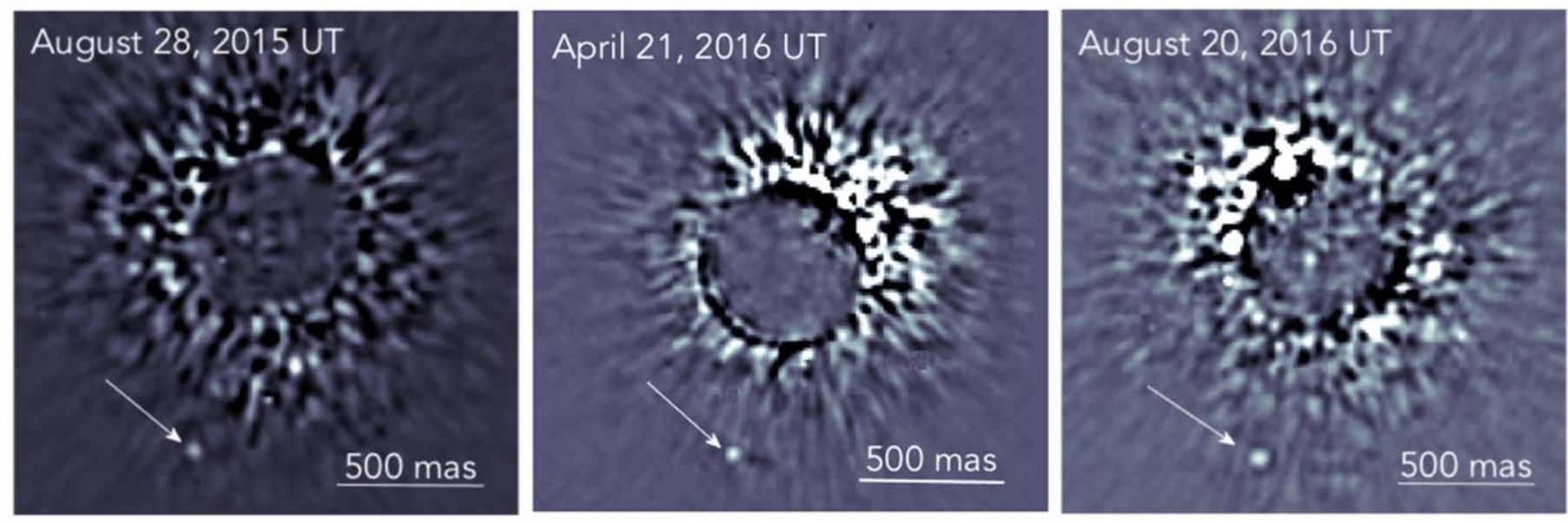

Figure 3. Images of HD 169889 B taken with NIRC2 in the $K_{s}$ band (left, right) and $H$ band (middle).

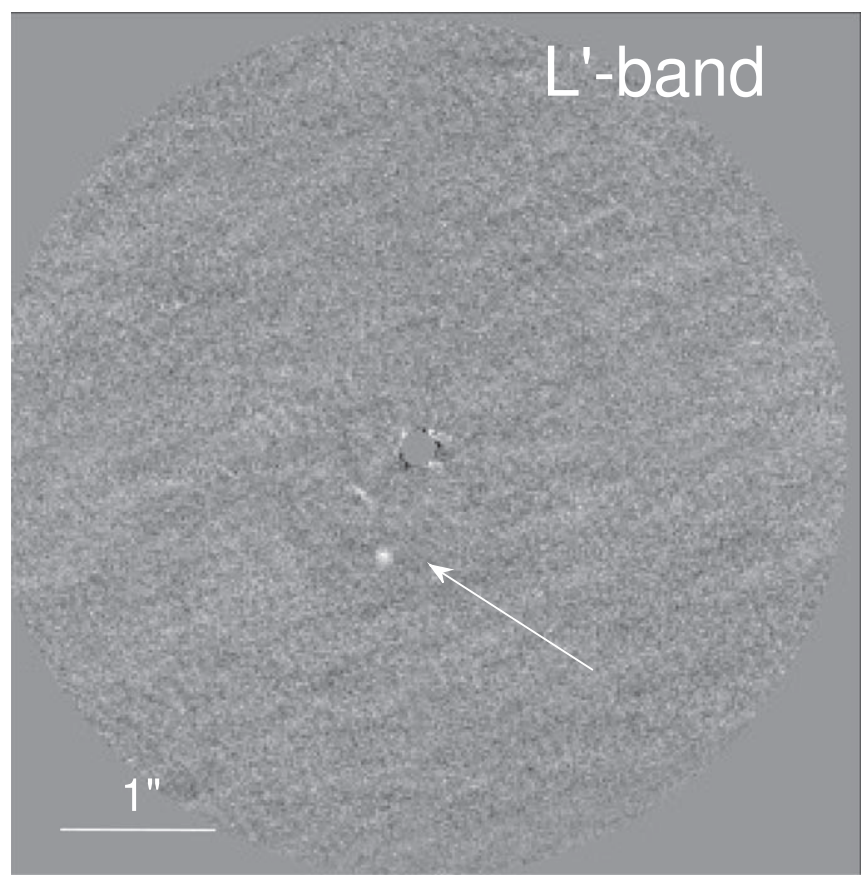

Figure 4. Confirmation image of HD 169889 B taken with LMIRCam at the LBT in 2016 June.

Table 2

Relative Stellar RV Measurements for HD 169889

\begin{tabular}{lccc}
\hline \hline $\begin{array}{l}\text { Date } \\
(\mathrm{UT})\end{array}$ & $\begin{array}{c}\text { BJD } \\
-2,450,000\end{array}$ & $\begin{array}{c}\mathrm{RV} \\
\left(\mathrm{m} \mathrm{s}^{-1}\right)\end{array}$ & $\begin{array}{c}\text { Uncertainty } \\
\left(\mathrm{m} \mathrm{s}^{-1}\right)\end{array}$ \\
\hline 2003 Jul 12 & 2832.8151 & -212.01 & 1.16 \\
2004 May 28 & 3154.0357 & -183.81 & 3.12 \\
\hline 2004 Aug 23 & 3240.8538 & -178.44 & 0.84 \\
2005 Jun 25 & 3546.8841 & -153.44 & 0.81 \\
2006 Sep 05 & 3983.7572 & -123.22 & 0.80 \\
2013 Aug 08 & 6512.7585 & 102.17 & 1.16 \\
2014 Aug 24 & 6893.7523 & 135.61 & 0.90 \\
2016 Jun 29 & 7568.8750 & 216.75 & 0.88 \\
\hline
\end{tabular}

Note. A horizontal line denotes the division between data sets when the HIRES detector was upgraded (2004 August) requiring a relative RV offset for analysis.
Table 3

Summary of High-contrast Imaging Observations Showing the Number of Coadds $\left(n_{\text {coadds }}\right)$, Frames $\left(N_{\text {fr }}\right)$, Exposure Time per Frame $\left(\Delta t_{\text {exp }}\right)$, and Change in Parallactic Angle $\Delta \pi\left(^{\circ}\right)$

\begin{tabular}{lcccc}
\hline \hline Date (UT) & Instrument & Filter & $\Delta t_{\exp } \times n_{\text {coadds }} \times N_{\text {fr }}$ & $\Delta \pi\left(^{\circ}\right)$ \\
\hline 2015 May 29 & NIRC2 & $K_{s}$ & $5 \times 10 \times 18$ & 1.8 \\
2015 Aug 28 & NIRC2 & $K_{s}$ & $5 \times 10 \times 30$ & 48.7 \\
2016 Apr 21 & NIRC2 & $H$ & $3 \times 15 \times 30$ & 10.5 \\
2016 Jun 20 & LMIRCam & $L^{\prime}$ & $0.08733 \times 1 \times 3948$ & 50 \\
2016 Aug 20 & NIRC2 & $K_{s}$ & $10 \times 3 \times 45$ & 20.5 \\
\hline
\end{tabular}

A more careful treatment would consider the slight curvature noticeable by eye in the RV time series as well as astrometry (Figure 1). However, only a small fraction of the companion orbit has been traced in either data set. Further, the astrometry measurements were obtained with two different instruments using three different filters. The systematics that result from combining these observations can be noticed in the zoomed-in portion of Figure 5 at the $\approx 1 \sigma$ level ( 10 mas). The WD companion appears to be moving to the north, i.e., closer to HD $169889 \mathrm{~A}$, but further AO imaging is required to confirm.

\section{WD Properties}

Empirical relations between WD masses and their progenitors have been established based on the study of open clusters, globular clusters, and common proper motion pairs (Weidemann 2000; Williams et al. 2009). Using the dynamical mass lower limit from RVs and imaging, we attempt to place a lower limit on the companion progenitor mass $\left(M_{i}\right)$ by inverting the empirical relationship from Catalán et al. (2008),

$$
M_{f}=(0.096 \pm 0.005) M_{i}+(0.429 \pm 0.015),
$$

which is applicable for $M_{i} \leqslant 2.7 M_{\odot}$ stars. ${ }^{12}$ We find, however, that the dynamical mass lower limit is smaller than all final mass $\left(M_{f}\right)$ values used to establish the empirical relation. Naively extrapolating the equation to smaller masses is not (justifiable nor) informative since the corresponding progenitor mass $\left(M_{i}\right)$ becomes smaller than the mass of HD 169889 A $\left(M_{i} \leqslant 0.89 M_{\odot}\right)$, which cannot be true provided that the pair formed at the same time.

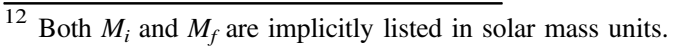


Table 4

Summary of Astrometry Measurements

\begin{tabular}{lcccc}
\hline \hline Date (UT) & JD-2,450,000 & $\rho$ (mas) & PA $\left(^{\circ}\right)$ & Proj. Sep. $($ au) \\
\hline 2015 May 29 & 7171.9 & $\approx 832 \pm 10$ & $\approx 163 \pm 1$ & $161.7 \pm 0.8$ \\
2015 Aug 28 & 7262.8 & $816 \pm 6$ & $162.1 \pm 0.7$ & $28.4 \pm 0.4$ \\
2016 Apr 21 & 7500.1 & $789 \pm 12$ & $162.0 \pm 0.5$ & $27.9 \pm 0.4$ \\
2016 Jun 20 & 7559.9 & $828 \pm 3$ & $161.5 \pm 0.7$ & $29.3 \pm 0.1$ \\
2016 Aug 20 & 7620.8 & $796 \pm 6$ & $28.1 \pm 0.2$ \\
\hline
\end{tabular}

Note. Position angle (PA) measurements are referenced with respect to the equator of the observing epoch.

Table 5

Photometric Results and Companion Properties

\begin{tabular}{ll}
\hline \hline & HD 169889 B \\
\hline$\Delta H$ & $9.76 \pm 0.16$ \\
$\Delta L^{\prime}$ & $9.60 \pm 0.03$ \\
$H$ & $16.32 \pm 0.16$ \\
$L^{\prime}$ & $16.05 \pm 0.08$ \\
$\mathrm{H}-\mathrm{L}$ & $0.27 \pm 0.18$ \\
$M_{H}$ & $13.59 \pm 0.16$ \\
$M_{L^{\prime}}\left(M_{\odot}\right)$ & $13.32 \pm 0.08$ \\
$M_{\text {dyn }}(\mathrm{Gyr})$ & $\geqslant 0.369 \pm 0.010$ \\
$t_{\text {cooling }}(\mathrm{g})$ & $\leqslant 5.2_{-1.5}^{+1.3}$ \\
\hline
\end{tabular}

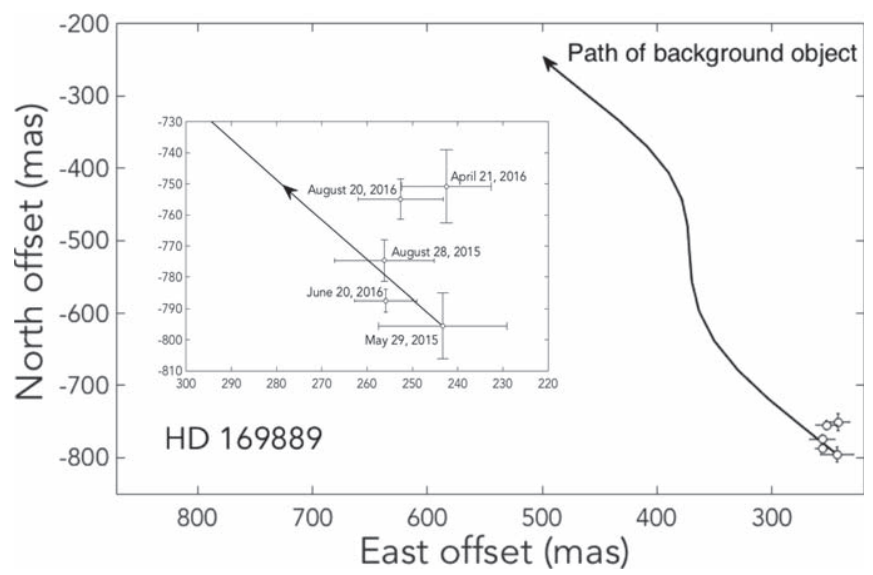

Figure 5. Proper motion and parallactic motion analysis taking into account the path that an infinitely distant background object would traverse is compared to the separation of HD $169889 \mathrm{~B}$ over time. Angular offsets are measured relative to the optical primary star. The inset shows a zoomed-in view of individual astrometric measurements. The companion shares the same space motion demonstrating that they are gravitationally associated.

We instead place a constraint on the nuclear-burning plus cooling age of the WD by estimating the age of HD 169889 A. $^{13}$ Using gyrochronology relations from Mamajek \& Hillenbrand (2008), we find $P_{\text {rot }}=33 \pm 4$ days, corresponding to a gyrochronological age of $\tau=5.2_{-1.5}^{+1.3} \mathrm{Gyr}$ (Wright et al. 2004). This estimate, which is based on the $B-V=0.75$ color of the primary (we assume \pm 0.01 uncertainty) and $R_{\mathrm{HK}}^{\prime}=-4.9$ value (we assume \pm 0.1 uncertainty), is consistent with the broad range suggested from an isochronal analysis (SME, Table 1).

To break the degeneracy between the nuclear-burning timescale and cooling age it is necessary to precisely determine the WD effective temperature (Liebert et al. 2005). With only two broadband photometry measurements available, we

\footnotetext{
13 Progenitor mass estimates are further complicated by chemical composition, e.g., whether the atmosphere is hydrogen-dominated, helium-rich, or a combination thereof.
}

consider the Rayleigh-Jeans tail of the Planck curve to get a handle on the WD effective temperature. Using the $H$ and $L^{\prime}$ measurements to obtain SED slope information, $d B_{\lambda} / d \lambda \approx\left(B_{L^{\prime}}-B_{H}\right) / \Delta \lambda$, we find a temperature of $T \approx 2150 \mathrm{~K}$. This value however would imply a blackbody peak in the near-infrared $\left(\lambda_{0} \approx 1.4 \mu \mathrm{m}\right)$ and is therefore inconsistent with the presumption that the WD is hot in the first place. We adopt $T=2150 \mathrm{~K}$ as a lower limit to the companion temperature, noting that WDs this cool have not been detected.

Knowing a priori that ultra-cool WDs are scarce, we fit a full blackbody curve to the SED by incorporating the distance to the source. We find that hotter temperatures, $T \approx 10,000 \mathrm{~K}$, are also consistent with the limited photometry available; however, large discrepancies still remain in the mid-infrared suggesting that the presence of hydrogen and helium may need to be accounted for to properly fit the data set.

Finally, we use the $H$-band absolute magnitude of HD $169889 \mathrm{~B}$ and compare to WD cooling models (see Holberg \& Bergeron 2006; Kowalski \& Saumon 2006; Bergeron et al. 2011; Tremblay et al. 2011). ${ }^{14}$ Masses in the range from $M=0.4-1.2 M_{\odot}$ are considered. The mass lower limit is matched to the lower limit from dynamics, and the mass upper limit corresponds to that available from theoretical evolutionary sequences. In this range, pure hydrogen atmospheres result in an effective temperature range of $\approx 4000-12,000 \mathrm{~K}$ with a cooling age of 5.3-1.9 Gyr, and pure helium atmospheres result in an effective temperature range of $\approx 4000-14,000 \mathrm{~K}$ with a cooling age of 5.8-1.3 Gyr. Given the gyrochronological age estimate of HD 169889 A of $\tau=5.2_{-1.5}^{+1.3} \mathrm{Gyr}$, we cannot at this point identify any tension with the models without further refinements to the companion mass. While these estimates are consistent with the blackbody temperature approximated using the H-L color, low-resolution spectroscopy is needed to further characterize the WD and understand its composition, cooling age, and progenitor mass.

\section{Concluding Remarks}

We have detected a WD companion that orbits a bright $(V=8.27)$ and well-characterized solar-type (G9 V) star with a precisely determined parallax $(28.297 \pm 0.066$ mas $)$. The census of these Sirius-like systems is known to be incomplete just beyond the solar neighborhood $(d \approx 25)$. The second WD discovery of the TRENDS high-contrast imaging program HD 169889 B offers a close-up view of a directly imaged compact object for which dynamical mass information is available.

We have estimated the nuclear-burning timescale plus cooling age of the WD using gyrochronology of

\footnotetext{
${ }^{14}$ http://www.astro.umontreal.ca/ bergeron/CoolingModels/
} 
HD 169889 A. At present, however, degeneracies between effective temperature, composition, and the companion final mass preclude an estimate of the compact object progenitor mass. Broader SED information along with continued Doppler and astrometric monitoring is warranted to further characterize the companion atmosphere and other physical properties. Space-based observations could provide complementary photometric and spectroscopic measurements and potentially allow for an estimate of $\log (g)$ through the Balmer line profile, and a gravitational redshift measurement would yield the WD massto-radius ratio provided that contamination from the bright optical host star can be mitigated. It may be worth investigating indirect evidence for past mass transfer from HD 169889 B to its optical host star through studies of enhanced metallicity in the optical spectrum. Finally, the companion will be valuable for characterizing high-contrast imaging spectrographs given its flux ratio and presumed featureless spectrum.

The TRENDS high-contrast imaging program is supported in part by NASA Origins of Solar Systems grant NNX13AB03G. J.R.C. acknowledges support from the NASA Early Career Fellowship and NSF Career Fellowship. E.J.G. acknowledges support from the NSF graduate research fellowship program. This research has made use of the SIMBAD database, operated at CDS, Strasbourg, France. Data presented herein were obtained at the W. M. Keck Observatory, which is operated as a scientific partnership among the California Institute of Technology, the University of California, and the National Aeronautics and Space Administration. The Observatory was made possible by the generous financial support of the W.M. Keck Foundation. The LBT is an international collaboration among institutions in the United States, Italy, and Germany. LBT Corporation partners are: The University of Arizona on behalf of the Arizona university system; Istituto Nazionale di Astrofisica, Italy; LBT Beteiligungsgesellschaft, Germany, representing the Max-Planck Society, the Astrophysical Institute Potsdam, and Heidelberg University; The Ohio State University, and The Research Corporation, on behalf of The University of Notre Dame, University of Minnesota, and University of Virginia. This work has made use of data from the European Space Agency (ESA) mission Gaia (https://www.cosmos.esa.int/ gaia), processed by the Gaia Data Processing and Analysis Consortium (DPAC, https://www.cosmos.esa.int/web/gaia/ dpac/consortium). Funding for the DPAC has been provided by national institutions, in particular the institutions participating in the Gaia Multilateral Agreement. This paper made use of WD cooling models from http://www.astro.umontreal.ca/ bergeron/ CoolingModels/. We are deeply grateful for the vision and support of the Potenziani and Wolfe families.

\section{ORCID iDs}

Justin R. Crepp (iD https://orcid.org/0000-0003-0800-0593

Brendan P. Bowler (iD https://orcid.org/0000-0003-2649-2288

Farisa Morales (D) https://orcid.org/0000-0001-9414-3851
Jordan Stone (iD https://orcid.org/0000-0003-0454-3718

Howard Isaacson (iD https://orcid.org/0000-0002-0531-1073

\section{References}

Amara, A., \& Quanz, S. P. 2012, MNRAS, 427, 948

Bacchus, E., Parry, I. R., Oppenheimer, R., et al. 2017, MNRAS, 469, 4796 Bergeron, P., Wesemael, F., Dufour, P., et al. 2011, ApJ, 737, 28

Bessell, M. S., \& Brett, J. M. 1988, PASP, 100, 1134

Bond, H. E., Bergeron, P., \& Bédard, A. 2017, ApJ, 848, 16

Brewer, J. M., Fischer, D. A., Valenti, J. A., \& Piskunov, N. 2016, ApJS, 225,32

Catalán, S., Isern, J., García-Berro, E., \& Ribas, I. 2008, MNRAS, 387, 1693

Crepp, J. R., Gonzales, E. J., Bechter, E. B., et al. 2016, ApJ, 831, 136

Crepp, J. R., Johnson, J. A., Fischer, D. A., et al. 2012b, ApJ, 751, 97

Crepp, J. R., Johnson, J. A., Howard, A. W., et al. 2012a, ApJ, 761, 39

Crepp, J. R., Johnson, J. A., Howard, A. W., et al. 2013, ApJ, 774, 1

Crepp, J. R., Johnson, J. A., Howard, A. W., et al. 2014, ApJ, 781, 29

Cutri, R. M., Skrutskie, M. F., van Dyk, S., et al. 2003, The IRSA 2MASS AllSky Point Source Catalog, NASA/IPAC Infrared Science Archive, http:// irsa.ipac.caltech.edu/applications/Gator/

Gaia Collaboration, Brown, A. G. A., Vallenari, A., et al. 2018, A\&A, 616, A1

Gaia Collaboration, Prusti, T., de Bruijne, J. H. J., et al. 2016, A\&A, 595, A1

Harris, H. C., Munn, J. A., Kilic, M., et al. 2006, AJ, 131, 571

Hinz, P. M., Defrère, D., Skemer, A., et al. 2016, Proc. SPIE, 9907, 990704

Holberg, J. B., \& Bergeron, P. 2006, AJ, 132, 1221

Holberg, J. B., Oswalt, T. D., Sion, E. M., Barstow, M. A., \& Burleigh, M. R. 2013, MNRAS, 435, 2077

Holberg, J. B., Oswalt, T. D., Sion, E. M., \& McCook, G. P. 2016, MNRAS, 462, 2295

Howard, A. W., Johnson, J. A., Marcy, G. W., et al. 2010a, ApJ, 721, 1467

Howard, A. W., Marcy, G. W., Johnson, J. A., et al. 2010b, Sci, 330, 653

Isaacson, H., \& Fischer, D. 2010, ApJ, 725, 875

Joyce, S. R. G., Barstow, M. A., Casewell, S. L., Holberg, J. B., \& Bond, H. E. 2017, in ASP Conf. Ser. 509, 20th European White Dwarf Workshop, ed.

P.-E. Tremblay, B. Gänsicke, \& T. Marsh (San Francisco, CA: ASP), 389

Kilic, M., Munn, J. A., Harris, H. C., et al. 2006, AJ, 131, 582

Kowalski, P. M., \& Saumon, D. 2006, ApJL, 651, L137

Liebert, J., Young, P. A., Arnett, D., Holberg, J. B., \& Williams, K. A. 2005, ApJL, 630, L69

Liu, M. C., Fischer, D. A., Graham, J. R., et al. 2002, ApJ, 571, 519

Maire, A.-L., Skemer, A. J., Hinz, P. M., et al. 2015, A\&A, 579, C2

Mamajek, E. E., \& Hillenbrand, L. A. 2008, ApJ, 687, 1264

Marois, C., Lafrenière, D., Doyon, R., Macintosh, B., \& Nadeau, D. 2006, ApJ, 641, 556

Matthews, C. T., Crepp, J. R., Skemer, A., et al. 2014, ApJL, 783, L25

Parsons, S. G., Gänsicke, B. T., Marsh, T. R., et al. 2017, MNRAS, 470, 4473

Parsons, S. G., Rebassa-Mansergas, A., Schreiber, M. R., et al. 2016, MNRAS, 463, 2125

Rodigas, T. J., Bergeron, P., Simon, A., et al. 2016, ApJ, 831, 177

Service, M., Lu, J. R., Campbell, R., et al. 2016, PASP, 128, 095004

Skemer, A. J., Hinz, P., Esposito, S., et al. 2014, Proc. SPIE, 9148, 91480L

Skrutskie, M. F., Cutri, R. M., Stiening, R., et al. 2006, AJ, 131, 1163

Skrutskie, M. F., Jones, T., Hinz, P., et al. 2010, Proc. SPIE, 7735, 77353H

Soummer, R., Pueyo, L., \& Larkin, J. 2012, ApJL, 755, L28

Tremblay, P.-E., Bergeron, P., \& Gianninas, A. 2011, ApJ, 730, 128

Valenti, J. A., \& Fischer, D. A. 2005, ApJS, 159, 141

van Winckel, H., Lloyd Evans, T., Briquet, M., et al. 2009, A\&A, 505, 1221

Vogt, S. S., Allen, S. L., Bigelow, B. C., et al. 1994, Proc. SPIE, 2198, 362

Weidemann, V. 2000, A\&A, 363, 647

Williams, K. A., Bolte, M., \& Koester, D. 2009, ApJ, 693, 355

Wizinowich, P., Acton, D. S., Shelton, C., et al. 2000, PASP, 112, 315

Wright, J. T., Marcy, G. W., Butler, R. P., \& Vogt, S. S. 2004, ApJS, 152, 261 